\title{
LBR-2 EARTH STATIONS FOR THE ACTS PROGRAM
}

\author{
Michael O'Reilly \\ Harris Corporation, Melbourne, FL \\ N91-11971. \\ Russell Jirberg and Ernie Spisz \\ NASA Lewis Research Center, Cleveland, $\mathrm{OH}$
}

\section{ABSTRACT}

Described in this paper is the "LBR-2" earth station being developed for NASA's Advanced Communications Technology Satellite (ACTS). The LBR-2 is one of two earth station types that operate through the satellite's baseband processor. The LBR-2 is a small VSAT-like earth station that is easily sited on a user's premises, and provides up to 1.792 megabits per second (MBPS) of voice, video, and data communications. Addressed in the paper is the design of the antenna, the rf subsystems, the digital processing equipment, and the user interface equipment.

\section{BACKGROUND}

NASA is currently developing two general classes of earth stations, an "LBR" class and an "HBR" class, for its Advanced Communications Technology Satellite (ACTS). The LBR variety operates through the satellite's Baseband Processor (BBP), whereas the HBR variety operates through its Microwave Switch Matrix (MSM). The basic system architecture for ACTS, as well as the details associated with the BBP and the MSM modes of operation have been described in earlier papers ${ }^{1,2,3}$.

For the BBP mode of operation NASA has defined two different types of LBR earth station: an LBR-1 and an LBR-2. In most respects the LBR earth stations are similar. Both are small VSAT-like earth stations that can be easily sited on the users' premises. Both operate in the ACTS' TDMA, demand-assigned multiple access (DAMA) environment. They differ only in terms of their throughput capacities and TDMA burst rates. The LBR-1 earth stations transmit at an uplink burst rate of $110 \mathrm{MBPS}$, and provide a total throughput capability of 46.528 MBPS. The LBR-1's are intended primarily for those users with a T3 (44.736 MBPS) requirement. The LBR-2's, on the other hand, are considered to be more of a general purpose earth station. They transmit at an uplink burst rate of $\mathbf{2 7 . 5}$ MBPS, and provide 1.792 MBPS of throughput capacity. This allows the LBR-2 earth station to meet the needs of those users whose requirements do not exceed that of a single T1 (1.544 MBPS). Described in this paper is the basic design that is being implemented for the LBR-2 earth station.

\section{DESIGN OVERVIEW}

Physically the LBR-2 earth station in many respects resembles the current generation of Ku-band VSAT's. It consists of an outdoor unit and an indoor unit as illustrated in Figure 1. Contained in the outdoor unit are the antenna, the receiver, and the transmitter. Contained in the indoor unit are the digital processing and control portions of the earth station, and the Terrestrial Interface Equipment (TIE) which is the interface to the user's telecommunications equipment.

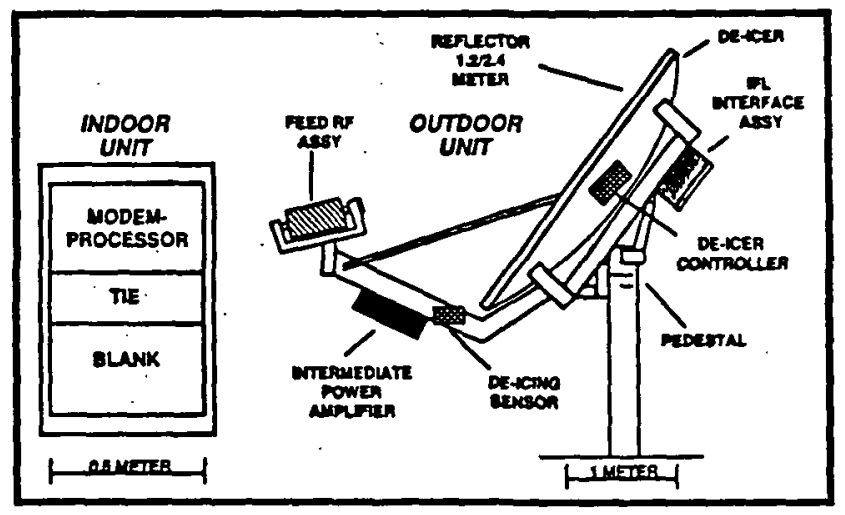

Figure 1. The LBR-2 Physical Configuration

The various subsystems that comprise the earth stations are indicated in Figure 2. The Modem. Processor performs a number of digital processing and control functions including multiplexing and demultiplexing of the uplink and downlink traffic, rate buffering, FEC encoding and decoding, and modulation and demodulation. It is also generates the various frequency and clock signals used throughout the earth station, and manages the various timing and control functions required to operate within the TDMA environment of the ACTS system.

The indoor and outdoor units are interconnected at IF. Uplink signals from the modulator are transferred to the upconverter located on the rear of the antenna using an IF of $700 \mathrm{MHz}$ Downlink 


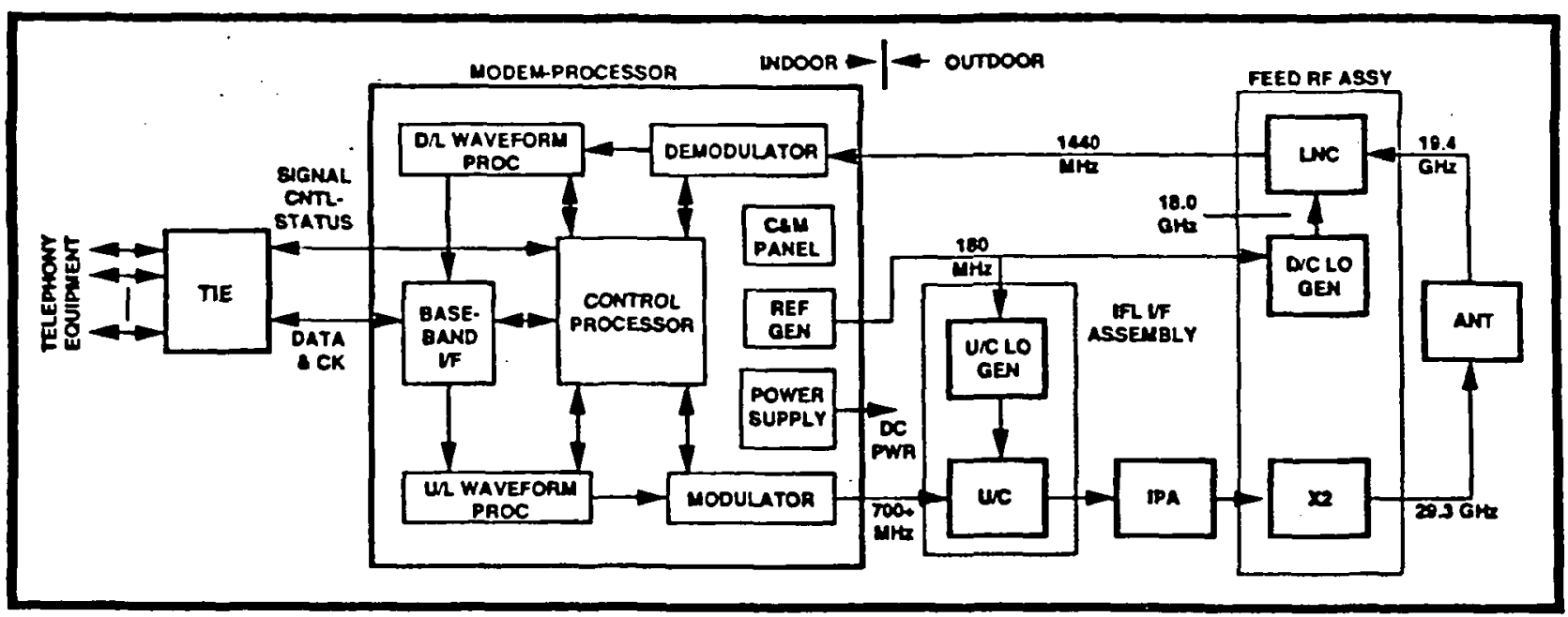

Figure 2. LBR-2 Functional Block Diagram

signals from the receiver, which along with thetransmitter is mounted on the feed support arm of the antenna, are transferred to the demodulator using an IF of $1440 \mathrm{MHz}$. The indoor-to-outdoor interconnect also includes a $180 \mathrm{MHz}$ reference frequency for the up and down frequency converters. Because of the relatively low IFs used for the interconnect, the indoor and outdoor units may be separated by 100 -foot long cable runs. For those installations requiring longer or shorter cable runs, amplifiers or pads may be used to boost or attenuate the signals accordingly.

\section{OUTDOOR UNIT}

\section{Antenna}

The LBR-2 earth station may be equipped with either one of two antennas: a 1.2-meter diameter antenna or a 2.4-meter diameter antenna. Both are offset prime focus designs with single-piece reflectors. This design was selected on the basis of its if performance and its reduced tendency for ice and snow buildup.

Both the 1.2-m and 2.4-m antennas are improved versions of an existing Ku-band VSAT design. Typically, reflectors designed for commercial Ku-band service will exhibit 15-20 mils of surface roughness. In a $40 \mathrm{mph}$ wind these antenna may deform by as much as 30 mils. At $30 \mathrm{GHz}$ surface deformities of this magnitude would result in an unacceptably large $3.8 \mathrm{~dB}$ loss of gain. Consequently, changes were made to the old design to improve the inherent surface accuracy of the reflector and to stiffen it structurally against wind induced deformities. Measurements made on a $2.4-\mathrm{m}$ protorype antenna indicate that the surface deformities in the new design due to simulated 40 $\mathrm{mph}$ wind loads were reduced to 13 mils. Hence, the wind induced losses in performance at $30 \mathrm{GHz}$ will be limited to an acceptable $0.7 \mathrm{~dB}$.

The reflector and its supporting structure, together with the feed supporn structure, are mounted on an EL-over-AZ positioner. The feed support structure is a tubular member that provides the primary support for the feed if assembly and for the transmitter. The If and power cables for the receiver and transmitter are routed through this tube. The feed rf assembly is mounted to the feed support structure by means of a feed saddle which allows three degrees of freedom of movement for focusing adjustments. The feed is located and aligned using three precision rods which are bolted to the reflector and feed saddle through precisely located alignment holes. Once aligned, two of the alignment rods remain an integral part of the assembly to stabilize the feed under windy conditions.

The antennas are designed to be mounted on a single vertical kingpost. For rooftop locations the antennas may be mounted on the non-penetrating platform shown in Figure 3. The mount is designed to maintain an azimuth and elevation pointing accuracy of $0.06^{\circ}$ under $40 \mathrm{mph}$ wind conditions. Ballast is provided by a set of concrete blocks which are contained in the four aluminum boxes. The boxes are bolted to the aluminum sandwich panels to eliminate unauthorized removal. Under $80 \mathrm{mph}$ winds this mount will limit the combined live and dead load of the larger 2.4-meter antenna to no more than 48.5 pounds per square foot. Tethers can be attached to contain the antenna in winds that exceed $80 \mathrm{mph}$. 


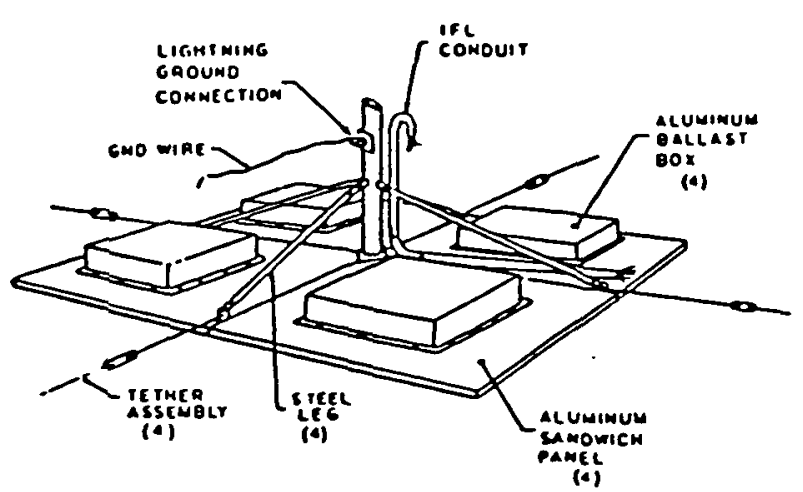

Figure 3. Non-Penetrating Roof Mount

Hydrophobic coatings will be used on the antennas as a rain repellent. Active de-icing in the form of radiant heating can be added to prevent ice and snow buildup. The heaters will be able to uniformly supply approximately 50 watts per square foot of radiant heating.

\section{Transmitter}

The transmitter design is unconventional in that it uses a Ku-band power amplifier driving a frequency doubler to generate the Ka-band uplink signal. To compensate for the bandwidth expansion that accompanies the frequency doubling, the signal generated by the modulator is first distorted by passing it through a divide-by-two circuit. After upconversion and amplification at Ku-band, the original modulation bandwidth is then regenerated by the doubler. Analysis, spectral measurements, and BER testing have shown that this $\div 2 / \times 2$ technique is a viable approach for SMSK modulation. The advantage of this approach is that it avoids the need for costly $30 \mathrm{GHz}$ upconverters and power amplifiers.
The basic design of the transmitter is indicated in Figure 4. It employs a Ku-band upconverter, a Kuband TWTA as an intermediate power amplifier (IPA), and a high power diode doubler as the final stage. The upconverter is a conventional design implemented with standard commercial components. The $13.860 \mathrm{GHz}$ local oscillator is phase-locked to the $180 \mathrm{MHz}$ reference frequency generated in the indoor unit.

The IPA is implemented using a conventional, commercially available unit. (This unit is currently in production for $\mathrm{Ku}$-band VSAT applications.) It contains a 60-watt TWT, a fault-tolerant power supply, and a controller. The entire unit is packaged in a compact, outdoor, weather-proof enclosure. An aircoldplate heat exchanger with dual redundant fans are used for cooling. Powered tests of this unit were successfully performed with the unit, including the fans, totally submerged in water. The IPA has the capability to deliver 50 watts of output power. With less than $\mathbf{4 0}$ watts required to drive the doubler, adequate design margins exists for end-of-life degradations.

The high-power frequency doubler utilizes a pair of epitaxially stacked varactor diodes. Similar frequency multipliers have been developed for EHF transmitter applications for use in a number of major military communications and radar programs. The simplicity of fabrication of these devices and their associated circuits make them ideally suited for commercial applications which require a simple, inexpensive means of generating millimeter-wave power. In addition, these devices have high projected reliabilities due to the fact that they operate with relatively high conversion efficiencies (diode efficiency $>75 \%$ ), and, hence, low junction temperatures. The median life expectancy of the complete doubler for the earth stations is estimated to be over 66,000 hours.

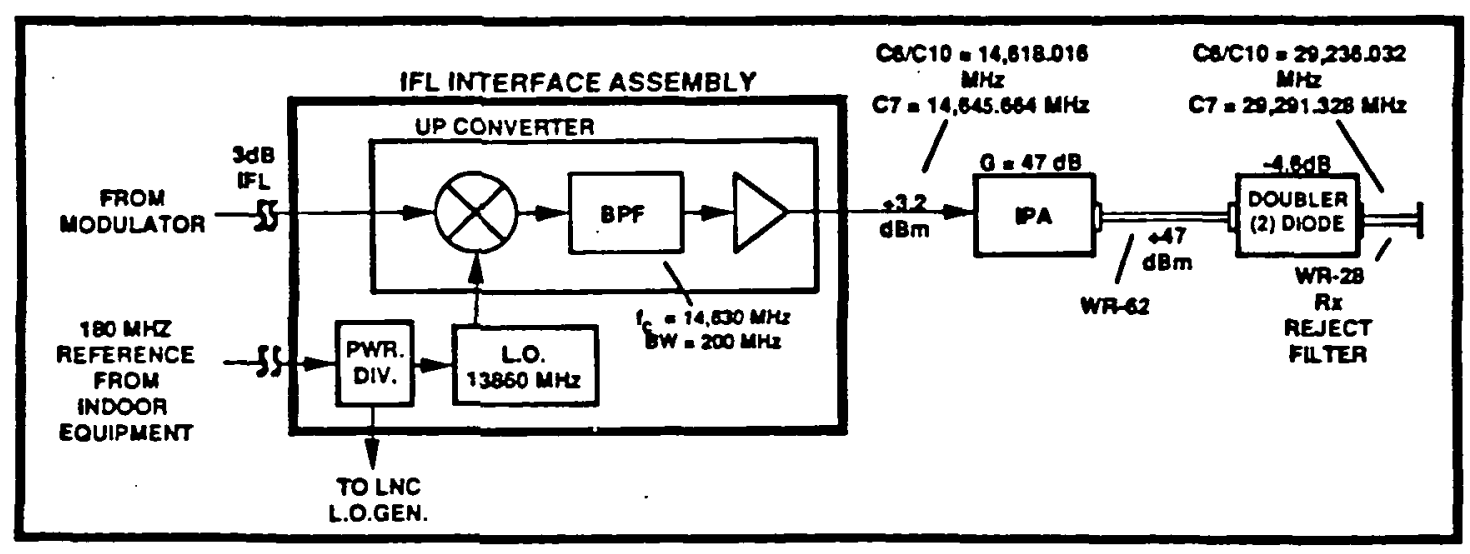

Figure 4. Transmitter Block Diagram 
The complete doubler consists of an input isolator followed by a power divider, two diode modules, a power combiner, and an output isolator. Preliminary tests indicated that the conversion efficiency of the doubler, including circuit losses, is $60 \%$. These $\mathrm{CW}$ tests also demonstrate that the two diode doubler approach is capable of producing the required 12 watts of output power.

\section{Receiver}

The receiver block diagram and downlink frequency plan are illustrated in Figure 5.

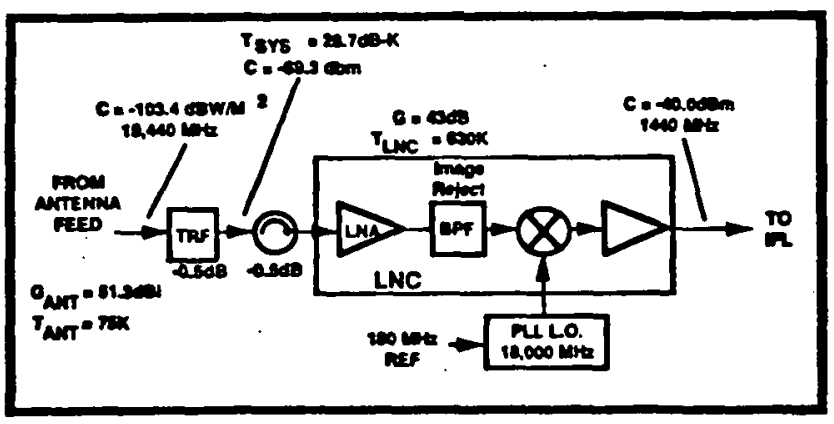

Figure 5. LBR-2 $19 \mathrm{GHz}$ Receiver

The transmit reject filter (TRF) together with the ortho-mode transducer (OMT) in the feed provide about $60 \mathrm{~dB}$ attenuation of the transmitted signal at the input to the low noise converter (LNC), thus eliminating any potential performance degradation to the LNC from the transmitter. The low noise amplifier (LNA) is a low cost design that uses packaged HEMT devices mounted on a single soft substrate. The IF amplifier is comprised of two low cost packaged Si MMIC devices. The $18 \mathrm{GHz}$ local oscillator is phase locked to the same $180 \mathrm{MHz}$ reference signal used by the upconverter. The TRF, the LNC, and local oscillator are all contained in the weatherproof feed if assembly which is located at the focal point of the antenna.
The LNA portion of the LNC has a gain of $23 \mathrm{~dB}$, with a demonstrated noise figure of $2.5 \mathrm{~dB}$ at room temperature. The $\mathrm{G} / \mathrm{T}$ performance of the earth stations is therefore estimated to be $16.1 \mathrm{dBi} / \mathrm{K}$ with the smaller $(1.2-\mathrm{m})$ antenna and $22.1 \mathrm{dBi} / \mathrm{K}$ with the larger (2.4-m) antenna.

\section{INDOOR UNIT}

The Modem Processor consisting of the modem, the digital processing equipment, the monitor and control equipment, the frequency sources, and the low voltage power supplies are all integrated within a single chassis. The Modem-Processor, together with the TIE, constitute the indoor unit. The entire unit is contained within a standard EIA 19-inch half-height equipment rack. Additional user equipment may be mounted in the space that remains in the equipment rack. The small size of the indoor unit allows it to be conveniently located within an office environment.

\section{Modulator}

The modulated uplink signal is generated using the implementation shown in Figure 6. The digital data from uplink rate buffer is first phase modulated, producing a BPSK spectrum centered at $630 \mathrm{MHz}$ The signal is then transformed into an SMSK signal centered at $636.575 \mathrm{MHz}$ by passing it through an offset conversion filter. A SAW filter is used to produce the $(\sin x) / x$ transfer function required for this conversion filter. The SMSK signal is then upconverted to one of two electrically selected frequencies, either $1,516 \mathrm{MHz}$ or $1,571 \mathrm{MHz}$, depending on which one of two uplink channels the station is assigned: $29,236.032 \mathrm{GHz}$ or $29,291.328 \mathrm{GHz}$. The filter bandwidths in the modulator and in the transmitter are sufficiently broad to pass either channel without the need for any switching or retuning of the filters.

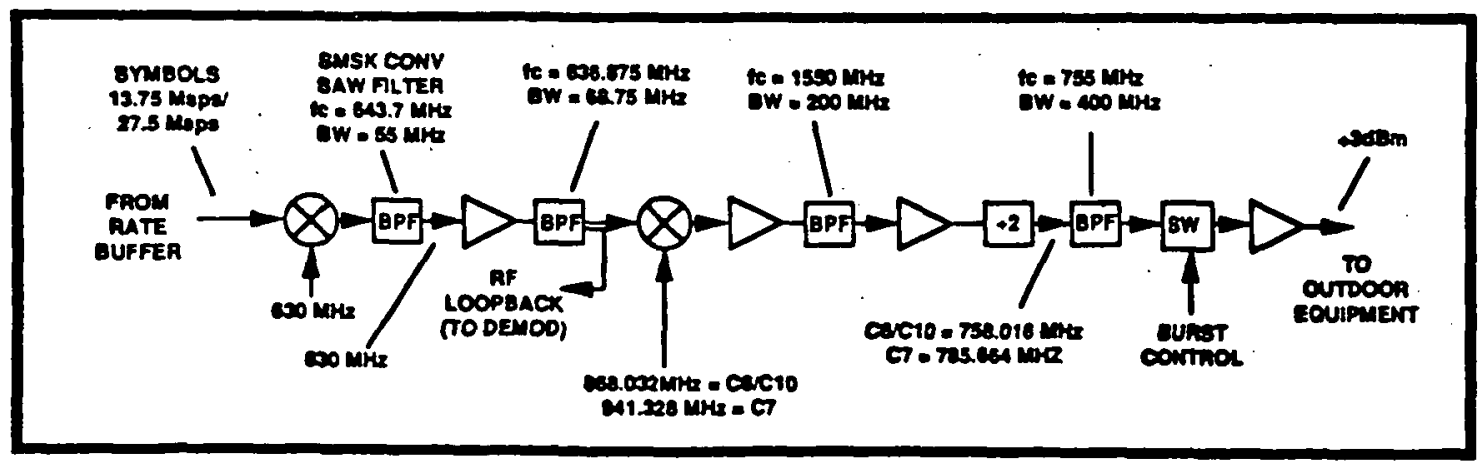

Figure 6. SMSK Modulator Block Diagram 
The modulated IF is then divided by 2 so that the signal produced by the frequency doubler in the transmitter will have the proper modulation index. Finally, the signal is passed through a pair of switches whose function is to create the TDMA uplink bursts. The signal emitted by the transmitter between bursts is attenuated by more than $50 \mathrm{~dB}$ by the action of the burst switch.

\section{Demodulator}

A block diagram of the demodulator is shown in Figure 7. The $1440 \mathrm{MHz}$ SMSK downlink signal from the outdoor unit is first downconverted to $630 \mathrm{MHz}$ The signal is then leveled by an AGC loop prior to the carrier recovery processing. The $A G C$ is designed to provide a dynamic signal range of $35 \mathrm{~dB}$ with level variations in excess of $0.5 \mathrm{~dB} / \mathrm{second}$.

The $630 \mathrm{MHz}$ IF signal is then split, one half going to the matched filter and the other half going to a frequency doubler. The matched filter is another SAW device which in this case is matched to the bit rate of the uncoded downlink signal (110.592 MBPS). The $1,260 \mathrm{MHz}$ output from the doubler is downconverted to $180 \mathrm{MHz}$, where the relatively narrow filter used for carrier recovery is realized. The output of the carrier recovery filter is then upconverted to $1,260 \mathrm{MHz}$ and applied to a divide-bytwo circuit. This produces a $630 \mathrm{MHz}$ coherent carrier for demodulating the signal obtained from the output of the matched filter.

The bit synchronizer utilizes two sampling analog. to-digital (AD) converters for data and clock recovery. These AVD converters are driven by two clocks that are $180^{\circ}$ out of phase, both of which are derived from the numerically controlled oscillator (NCO). The AVD converter in the data path samples the eye pattern at its point of maximum opening where little intersymbol interference exists. The AND converter in the clock recovery path samples the eye pattern at the zero crossings. The clock recovery $\mathrm{A} / \mathrm{D}$ in combination with the loop filter function as a timing error detector that updates the NCO at every bit transition. Since the downlink burst rate varies by no more than 1 part in $10^{5}$, the burst-to-burst frequency error will be very small. Consequently, the corrections made to the NCO, which is locked to the reference oscillator in the earth station, effectively adjust only the phase of the clock. The performance of this demodulator is within $1.5 \mathrm{~dB}$ of ideal.

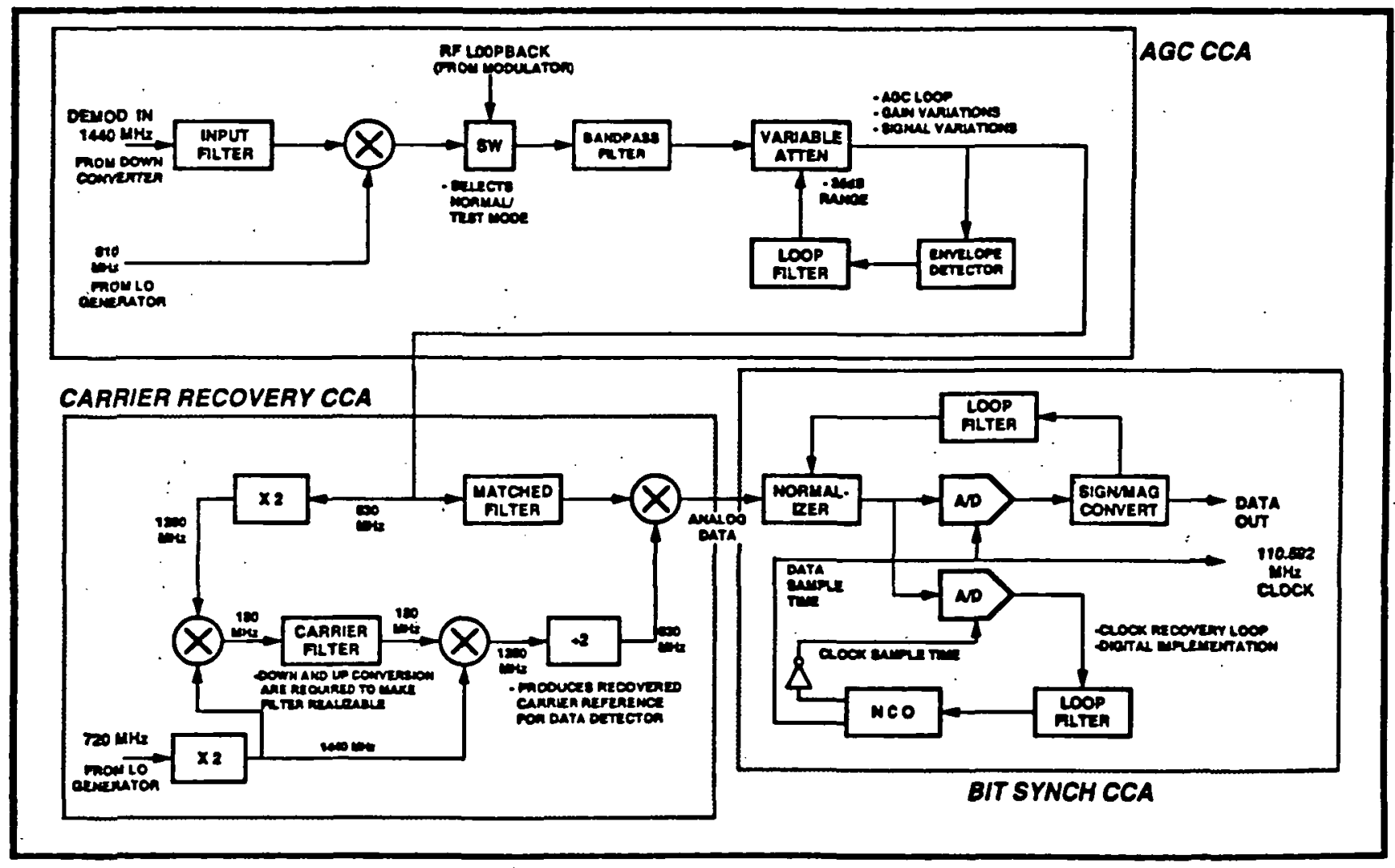

Figure 7. The LBR-2 Demodulator Block Diagram 


\section{FEC Codec}

Under rain fade conditions the BER can be maintained below $5 \times 10^{-7}$ by the combined effect of a 2:1 reduction in the burst rate and the application of forward error correction (FEC) coding. Using a rate $1 / 2$, constraint length 5 , convolutional code, ACTS can provide approximately $10 \mathrm{~dB}$ of rain fade protection. The FEC coding is applied adaptively only to those uplinks and downlinks requiring the added protection. In general, the uplinks and downlinks may be independently coded. However, for the LBR-2 earth stations, the FEC coding is always applied simultaneously to both links.

The decoder used in the Modem-Processor is a single chip, $1.5 \mu \mathrm{m}$ CMOS gate array. It implements a Viterbi (maximum likelihood) decoding algorithm for the rate $1 / 2$ convolutional code used by ACTS. The encoder is also a single chip device. It, along with the uplink data scrambler, share a common programmable logic array. (Data scrambling is used to maintain the bit transition density of the uplinks and downlinks at or near $50 \%$.)

\section{IDMA Processor}

Indicated in Figure 8 are the TDMA processing and control functions performed by the ModemProcessor. The signal from the demodulator (upper right) is first examined by the unique word detector which senses the unique word embedded in the downlink preamble. (The unique word is used to establish 64-bit word synchronization.) The signal then is rate buffered down to 6.912 MBPS, decoded (if necessary), and descrambled. At this point the outbound orderwire (OBOW) messages from the Master Control Station (MCS) and from other earth stations are demultiplexed and routed to the control processor. The remaining message trafic is then buffered in an elastic buffer and routed to the TIE.

On the uplink, the user traffic from the TIE is buffered in an elastic buffer and then multiplexed together with the inbound orderwire (IBOW) messages from the control processor. The message data is then scrambled and, if necessary, FEC encoded. An uplink preamble is then attached to the message data. The entire message is then rate buffered up to either the 27.648 MBPS rate used for clear-sky (i.e. uncoded) operations, or to the 13.824 MBPS rate used during rain fades.

All TDMA timing and control functions are managed by a single microprocessor. Stored programs automatically perform the following functions without the need for operator intervention:

- station initialization and status

- IBOW and OBOW processing

- U/L and $D / L$ frame processing

- burst time plan management

- TIE signaling and control

- rain fade sensing and control

- fault isolation

- man/machine interface

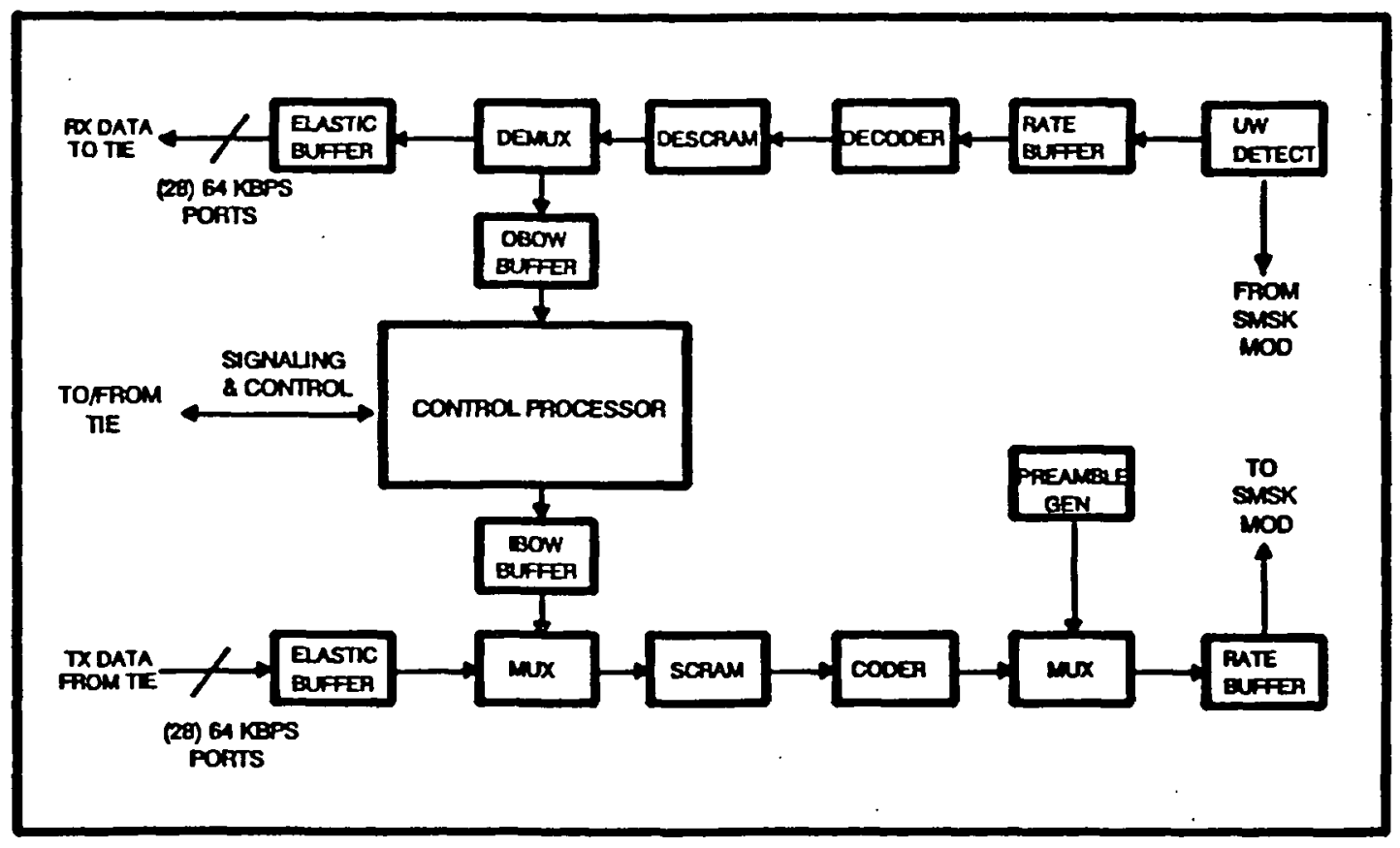

Figure 8. TDMA Processing Flow 
Separate foreground and background control maps are used to manage the network burst time plans established by the MCS. In this way the control processor is able to maintain an uninterrupted flow of traffic across the burst time plan change boundaries.

The control processor is also responsible for monitoring the performance of the earth station. Built into the earth station are a number of sensors, stimulators, and test loops which the control processor continuously monitors to check the health of the earth station and to isolate faults. The operating conditions are displayed on a front panel mounted readout as illustrated in Figure 9. A $4 \times 40$ character alphanumeric display is used to indicate the station's operating mode, its health status, the occurrence of any on-line fault indications, and the link quality status. In the off-line fault isolation mode, the appropriate failure indication is displayed.

In order to maintain uplink synchronization, the earth station must have an accurate internal timing source that is stable to \pm 1 part in $10^{9}$. Contained in the Modem-Processor is a small $10 \mathrm{MHz}$ rubidium cell atomic clock. All timing signals and frequency sources are derived from that clock. The rubidium cell is not only a cost effective means of providing a stable reference, it has the distinct advantage of enabling the earth stations to operate in excess of two years without recalibration.

\section{Rain Fade Control}

Link degradations due to rain fades are detected by sensing the $E_{b} / N_{0}$ of the downlink traffic-bearing signal. This method of fade detection has three advantages: (1) it avoids the expense of a beacon receiver, (2) it allows the earth station to be operated outside the coverage area of the beacons, and (3) it allows the system to automatically compensate for equipment degradations that affect the station's $G / T$ performance.

The method is based on a statistical measurement of the signal plus noise. The $E_{b} / N_{0}$ of the downlink signal is estimated by determining the ratio of the mean $\left(\mu^{2}\right)$ to the variance $\left(\mathrm{s}^{2}\right)$ of the signal. Analysis indicates that the measurement will be accurate to within $\pm 0.6 \mathrm{~dB}$ over the required $10 \mathrm{~dB}$ dynamic fade range. The computations required to determine $E_{b} / N_{0}$ are done by the control computer in the ModemProcessor. The fade status is automatically communicated to the MCS by means of the inbound orderwire. The decision to invoke and remove FEC coding may be done either at the earth station or by the MCS. The fade thresholds for invoking and removing FEC coding at the onset and cession of a fade event may be adjusted so as to adapt the station to its specific rain fade environment.

For the purposes of experimentation, attenuators may be inserted in the transmitter and receiver to independently restrict the earth station's clear-sky uplink margin and downlink margin. This will allow the speed and effectiveness of the rain fade control algorithms to be evaluated.

\section{TERRESTRIAL INTERFACE}

The TIE (Figure 10) is connected to the ModemProcessor by means of 28 duplex synchronous data lines operating at $64 \mathrm{KBPS}$. Clock rate difference between terrestrial circuits and the space segment are absorbed by elastic (plesiochronous) buffers at the input and output ports to the TDMA Processor. A 2400 baud asynchronous data link is used to convey signaling and control information between the user and the Modem-Processor.

The TIE is a commercial piece of telecommunications equipment. It is equipped with a number of field-changeable modules that will enable the user to configure the interface to meet his particular set of requirements for voice, video, and data services. The user may connect any one or more of the following:

- two-wire analog telephones

- ground/loop start lines and trunks

- two-wire and four-wire E\&M trunks

- T1 (DS-1) digital lines

- synchronous serial data (8 - 64 KBPS)

- asynchronous serial data (300 - 9600 baud)

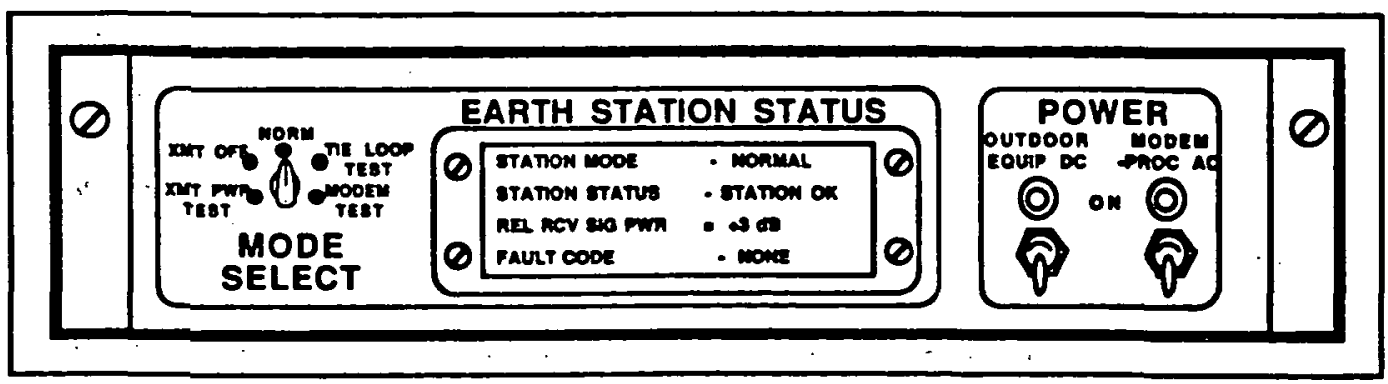

Figure 9. The Control and Monitor Panel 
The mix of circuit types is limited only by the throughput capacity of the station (1.792 MBPS). The user may therefore, for example, connect one full T1 (1.544 MBPS) circuit plus four DS-0 (64 KBPS) circuits, or any other equivalent combination of 28 DS-0 circuits. The electrical and signaling protocols for the various interfaces adhere to the conventional Bell standards.

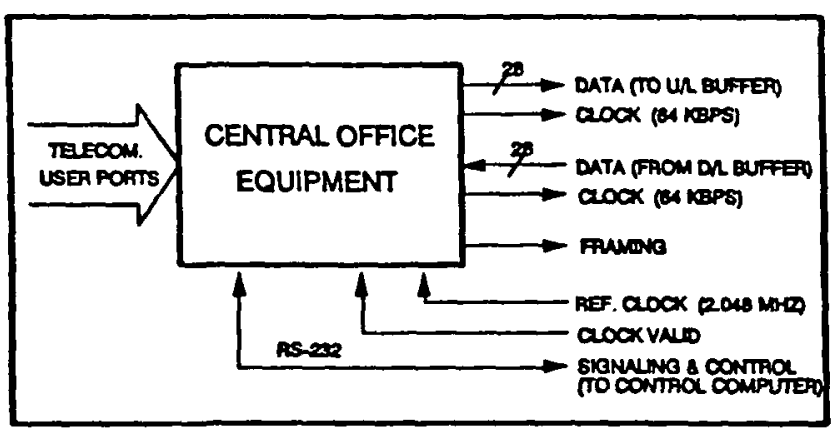

Figure 10. Terrestrial Interface Equipment

Functionally the TIE, in conjunction with the signaling and control software that resides in the control computer of the Modem.Processor, is equivalent to a local telephone central office. Call set-ups and tear-downs are managed using the customary DTMF signaling tones, call progress tones, and cadences. The signaling that takes place between the earth station and the MCS is totally transparent to the user.

All circuits connected to the TIE do not necessarily have to be routed over the satellite. The earth station has the capability to interconnect circuits locally in the same manner as commercial central offices do. This gives the earth station the flexibility to function as a true communications center.

\section{INSTALLATION}

The earth station is specifically designed to facilitate its installation on users' premises. It is shipped partially disassembled, the largest single piece being the 2.4-meter reflector. Once on site, the earth station can be reassembled, installed, and checked out by two installers. Two signals that are generated in the indoor unit are cabled out to the antenna: a downlink lock indicator and an analog AGC signal. These signals provide a measure of the received signal strength to assist the installers in aligning the antenna. With the proper site preparation, the installation can be completed in less than 8 hours.

\section{RELIABILITY}

The mean-time-between-failures (MTBF) of the earth station is projected to be in excess of 2,200 hours. The self-test capability built into the earth station should allow over $90 \%$ of all equipment failures to be detected and isolated. Because of the modular construction of the earth station, the meantime-to-repair (MTTR) is estimated to be less than one hour.

To eliminate failures due to infant mortality, each earth station will be thermally stress tested by repeatedly cycling the equipment between $0^{\circ} \mathrm{C}$ and $55^{\circ} \mathrm{C}$ with power on. Following the thermal stress test, each earth station will be run for a minimum of 200 consecutive failure-free hours. Experience has shown that this type of testing reduces the failure rate of equipment in the first two years of its operation by as much as $50 \%$.

\section{REFERENCES}

1. J. C. Graebner and W. F. Cashman, "Advanced Communications Technology Satellite: System Description," IEEE Global Communications Conference, Vol. 1, pp. 559-567, Dec. 1986.

2. F. M. Naderi and S. J. Campanella, "NASA's Advanced Communications Technology Satellite (ACTS): An Overview of the Satellite, the Network, and the Underlying Technologies, "AlAA 12th International Communications Conference, Arlington, Virginia, March 1988.

3. T. Inuaki, D. Juplin, R. Lindstrom, D. Meadows, "ACTS TDMA Network Control Architecture," Proceedings 12th International AIAA Communications Satellite System's Conference, March 1988, Washington, D.C. 